\title{
Influence of oxygen on surface morphology of metalorganic vapor phase epitaxy grown GaAs (001)
}

\author{
S. Nayak, ${ }^{a}$ J. W. Huang, J. M. Redwing, D. E. Savage, M. G. Lagally, and T. F. Kuech \\ University of Wisconsin, Madison, Wisconsin 53706
}

(Received 6 December 1995; accepted for publication 17 December 1995)

\begin{abstract}
Atomic force microscopy has been used to investigate the influence of controlled oxygen incorporation on the surface morphology of GaAs films grown by metalorganic vapor phase epitaxy (MOVPE). Oxygen influences the periodic morphology observed in GaAs surfaces, with concentrations about $10^{18} \mathrm{~cm}^{-3}$ leading to a breakup of the periodicity. To account for these observations, we propose a model in which oxygen preferentially attaches at steps with a subsequent reduction in step mobility and a concomitant increase in the surface roughness. (C) 1996 American Institute of Physics. [S0003-6951(96)04608-9]
\end{abstract}

GaAs grown by metalorganic vapor phase epitaxy (MOVPE) has been used extensively in electronic and photonic devices. Despite the wide applications of MOVPE GaAs materials, a fundamental understanding of the growth process is still currently being developed. Many studies have been directed toward understanding the basic MOVPE growth chemistry, ${ }^{1-4}$ but only a few address the growth mechanisms at the atomic scale. In particular, very little is known about the impact of impurities on the growth mechanisms and evolution of growth front morphology.

The growth front morphology of molecular-beam epitaxy (MBE)-grown GaAs has been investigated by scanning tunneling microscopy, ${ }^{5,6}$ atomic force microscopy $(\mathrm{AFM}),{ }^{7,8}$ and reflection high-energy electron diffraction. ${ }^{5,7}$ A periodic or quasiperiodic morphology consisting of elongated mounds is generally observed. Mound morphology in growing films has been variously ascribed to thermodynamic driving forces or kinetic limitations. Both could in principle, be true. Thermodynamic arguments ${ }^{6}$ demand that the growth front adopts a morphology that minimizes the free energy of the surface: anisotropic step energies can lead to anisotropic shapes and steps may bunch to form specific lower-energy facets, especially in systems that involve strain. Kinetic arguments ${ }^{6,8}$ rest $^{-}$ on limited diffusion rates, anisotropy in diffusion, and especially the existence of barriers for atomic transport over steps. These factors cause the development of periodic three dimensional (3D) morphology. ${ }^{9-11}$

Although MOVPE growth is qualitatively different from MBE growth, similar fundamental thermodynamic driving forces and kinetic limitations must control the growth front morphology. Periodic growth front morphology has also been observed in MOVPE-grown GaAs(001) ${ }^{1-4}$ All work reported on growth front morphology in MOVPE grown GaAs has so far, focused on growth without deliberate incorporation of impurities. In this letter, we report on the evolution of growth front morphology in GaAs (001) using atomic force microscopy when there is such incorporation. We show that the concentration of the impurity, here oxygen, affects the period and height of the features formed at the growth front. We present a model in which the evolving morphology is controlled by a modification of the kinetic parameters by the impurity.

${ }^{a)}$ Electronic mail: nayak@mregcvd.engr.wisc.edu
Oxygen is a common, often inadvertent impurity in MOVPE growth environments. ${ }^{12,13}$ Oxygen has been suggested to initiate bulk morphological defects during growth but its specific influence on surface morphology of epitaxial GaAs has not been addressed on an atomic scale. To begin to address this issue, we have made AFM measurements of the morphology of the growth front of GaAs (001) films intentionally doped with various concentrations of oxygen during growth.

Epitaxial films were grown on semi-insulating and $n$-type GaAs (001) substrates with a miscut angle of $1.95^{\circ}$ $\pm 0.1^{\circ}$ toward [010]. In GaAs (001) miscut in this direction, a step will consist of an equal amount of A- and B-type segments, which are, respectively, Ga and As terminated. Epitaxial GaAs films were grown at $600{ }^{\circ} \mathrm{C}$ using trimethylgallium and arsine at a growth rate of $3 \mu \mathrm{m} / \mathrm{h}$. Details of the oxygen incorporation in GaAs using diethylaluminum ethoxide (DEALO) $\left[\left(\mathrm{C}_{2} \mathrm{H}_{5}\right)_{2} \quad \mathrm{AlO} \quad \mathrm{C}_{2} \mathrm{H}_{5}\right]$ are discussed elsewhere. ${ }^{13}$ The oxygen concentration was controlled between $10^{16}$ and $10^{20} \mathrm{~cm}^{-3}$. The sample structure consisted of an undoped buffer layer of GaAs, $0.1 \mu \mathrm{m}$ thick, followed by $5 \mu \mathrm{m}$ of undoped or oxygen doped GaAs. The average oxygen concentration in the film was determined by secondary ion mass spectrometry (SIMS). The surface morphology was investigated by AFM in a constant-force mode.

Figure 1 shows images obtained from a $1 \times 1 \mu \mathrm{m}^{2}$ area as a function of doping concentration. The surface morphology of nominally undoped $5 \mu \mathrm{m}$ thick GaAs (001) [Fig. 1(a)] exhibits 1D periodic structure along [010], the miscut direction of the substrate, and is independent of doping. No periodicity is evident in the orthogonal direction. The root mean square (rms) roughness $(\sigma), \sigma=\sqrt{\left\langle[h(r)-\bar{h}]^{2}\right\rangle}$, where $\bar{h}$ is the mean height of the surface and $h(r)$ is the height of the surface at a distance $r$ on the surface, was calculated from AFM images. One-dimensional spectral analysis of data was performed along [010] and averaged over 128 lines, to evaluate the contribution of various sinusoidal waves to the periodic morphology of the surface. The wavelengths $(\lambda)$ of the sinusoidal wave with the maximum intensity $\left(A^{2}\right)$ and the range of wavelengths corresponding to half the maximum intensity were calculated by fitting a curve to the spectrum. Table I summarizes the values of $\sigma, \lambda$, and $A$.

The spatial wavelength of the morphologies $(\lambda)$, which is always much greater than the nominal step separation $(\sim 8$ 

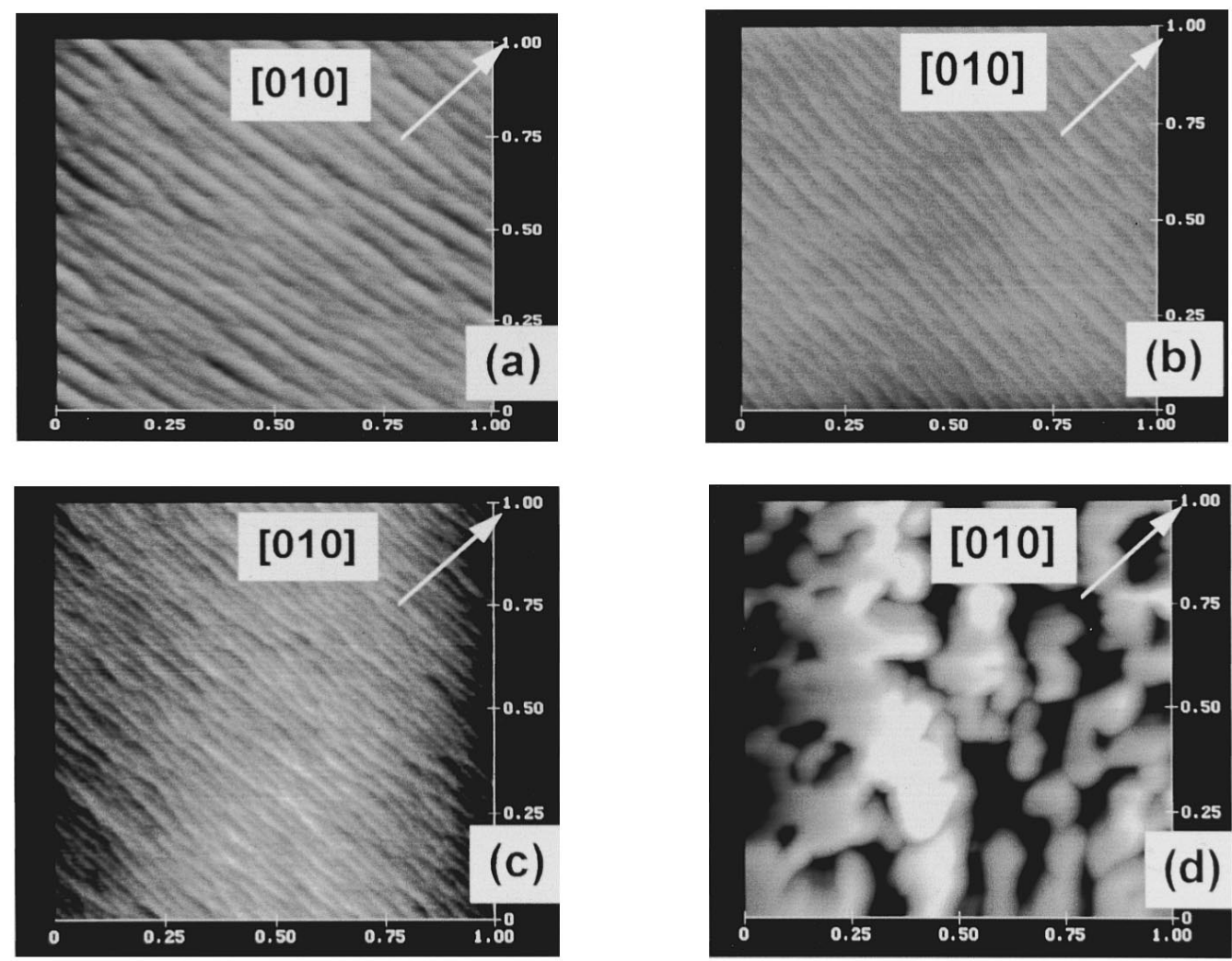

FIG. 1. $1 \mu \mathrm{m} \times 1 \mu \mathrm{m}$ AFM images of oxygen doped GaAs; (a) nominally undoped, (b) $10^{16}$, (c) $10^{18}$, and (d) $10^{20} \mathrm{~cm}^{-3}$. A periodic ripple structure in the miscut direction is established through step bunching for all but the highest doping levels. The ripple wavelength decreases as [O] increases. The 3D-cluster growth takes place at highest doping.

$\mathrm{nm}$ ) of the substrate, decreases with increasing "bulk oxygen concentration" up to $[\mathrm{O}] \sim 10^{18} \mathrm{~cm}^{-3}$. At bulk oxygen concentrations above $10^{18} \mathrm{~cm}^{-3}$, a breakdown of periodic morphological features is evident, indicating a disruption in the previous mode of epitaxial growth. An islandlike structure, as shown in Fig. 1(d), evolves.

The three principal observations of this study are: (1) the miscut direction and feature directions are the same, in agreement with other MOVPE studies; ${ }^{1-3}$ (2) although the qualitative features of the surface morphology are not affected by a bulk $\mathrm{O}$ concentration of less than $10^{19} \mathrm{~cm}^{-3}$ quantitatively the spatial period of the surface morphology decreases and the rms value of the roughness increases as the $\mathrm{O}$ concentration increases; and (3) at bulk concentrations about $10^{19} \mathrm{~cm}^{-3}$ a new surface morphology, consisting of $3 \mathrm{D}$ clusters rather than ridges evolves.

Before suggesting a model to explain the observed behavior, we briefly evaluate the existing data in terms of pos- sible thermodynamic or kinetic mechanisms. Both thermodynamic and kinetic arguments have been invoked ${ }^{1-8}$ In all the MOVPE data, ${ }^{1-4}$ including ours, the periodic morphology is aligned along whatever direction is chosen for the miscut. Although thermodynamic arguments can be made for stability of steps with a given orientation, it is difficult to make such arguments if the periodic morphology is always aligned with the miscut direction.

Kinetic limitations are much more credible causes for large-scale periodic morphology. On a very flat surface the nucleation is homogeneous in the sense that atoms must find each other on a terrace to form 2D islands. Islands separated by more than a diffusion length will be stable. If there subsequently exists a barrier for the downward motion over the island edges of atoms arriving on top of 2D islands, then a 3D "mound" morphology will evolve. If anisotropy in diffusion of atoms exists, then the mound structure is elongated along the fast diffusion direction and may form a periodic or

TABLE I. Surface rms roughness $(\sigma$ in $\mathrm{nm})$ and wavelength of periodic $(\lambda$ in $\mathrm{nm})$ surface structure in $1 \mu \mathrm{m}$ thick $\operatorname{GaAs}(001)$ with several concentrations of $\mathrm{O}$ are shown. The $\lambda$ and amplitude $(A$ in $\mathrm{nm})$ are shown up to $10^{18} \mathrm{~cm}^{-3}[\mathrm{O}]$ in GaAs. The breakdown of periodic structure at higher oxygen concentrations makes analysis not meaningful there.

\begin{tabular}{|c|c|c|c|c|c|c|}
\hline & \multicolumn{5}{|c|}{$[\mathrm{O}]$ in $\mathrm{GaAs}\left(\mathrm{cm}^{-3}\right)$} & \multirow{2}{*}{$\begin{array}{c}\text { Nominally } \\
\text { undoped }\end{array}$} \\
\hline & $10^{20}$ & $2.6 \times 10^{19}$ & $3 \times 10^{18}$ & $2.6 \times 10^{17}$ & $2 \times 10^{16}$ & \\
\hline$\sigma(1 \mu \mathrm{m} \times 1 \mu \mathrm{m})$ & $37.2 \pm 10$ & $5.91 \pm 1.0$ & $0.98 \pm 0.1$ & $0.58 \pm 0.1$ & $0.55 \pm 0.1$ & $0.62 \pm 0.1$ \\
\hline$\sigma(5 \mu \mathrm{m} \times 5 \mu \mathrm{m})$ & $56.7 \pm 10$ & $24.19 \pm 10$ & $2.09 \pm 1.0$ & $1.21 \pm 0.2$ & $0.90 \pm 0.2$ & $0.76 \pm 0.1$ \\
\hline$\lambda$ at $\max . \mathrm{A}^{2}$ & & & 30.8 & 40.6 & 46.1 & 56.2 \\
\hline $\max . \mathrm{A}$ & & & 1.41 & 1.73 & 1.90 & 2.21 \\
\hline$\lambda$ at $\left(\mathrm{A}^{2} / 2\right)$ & & & $25-44$ & $32-60$ & $38-55$ & $44-72$ \\
\hline
\end{tabular}


quasiperiodic structure along the slower diffusion direction. If steps are present in the substrate, the length over which atoms can move without crossing a step will be limited, increasing the nucleation density of mounds and producing a mound period smaller than that on a flat surface whenever a barrier for crossing the steps exists. The morphology will be anisotropic, because, in the direction in which there are no steps, the diffusion length will continue to be higher and the separation of mounds will therefore be similar to that on a flat surface. For typical miscuts, the step spacing is much smaller than the diffusion distance. The mound morphology will therefore form along the miscut direction, which here, is [010]. The magnitude of the kinetic barrier for crossing the steps will determine the mound periodicity.

Kinetic barriers at steps are the probable cause of periodic morphology in all these experiments. ${ }^{11}$ We are not aware of either MBE or MOVPE studies in which the deposition rate or substrate temperature were changed sufficiently to control the initial nucleation and therefore, the density of periodic mounds. However, the same result can be achieved with impurity incorporation during growth. If the impurity adsorbs at the steps and acts to modify the barrier for transport of atoms across a step or pins the steps, an increase in impurity concentration will lead to reduced diffusion and increased bunching.

Oxygen can induce this effect in GaAs. If we assume that at any instance the surface concentration of $\mathrm{O}$ during growth is given by the subsequently measured bulk concentration and that all the oxygen is incorporated at the steps, on a $2^{\circ}$ misoriented GaAs $(001)$ substrate, for $[\mathrm{O}] \approx 10^{18}$ $\mathrm{cm}^{-3}$, one in 80 step sites would be associated with an oxygen atom for each ML of GaAs that is deposited. The question may arise whether oxygen segregates to the GaAs surface during growth. Even though oxygen has a very low equilibrium solid solubility $\left(\mathrm{GaAs}: \mathrm{O}<10^{16} \mathrm{~cm}^{-3}\right)^{14}$ in $\mathrm{GaAs}$, there are no reports of surface segregation or precipitation of oxygen in MOVPE grown GaAs above the solubility limit. Dopants in MOVPE and MBE have been incorporated as isolated impurities at concentrations well beyond the solid solubility limit. With regard to the assumption that $\mathrm{O}$ incorporated at the steps, in a separate study of oxygen incorporation in MOVPE-grown GaAs, we have found that oxygen attaches preferentially at Ga terminated steps relative to As terminated steps. ${ }^{15}$ A strong metal-O bond, which is enhanced with our $\mathrm{O}-\mathrm{Al}$ precursor, ${ }^{13}$ will furthermore, suppress oxygen segregation at the growth front. We therefore, believe that $\mathrm{O}$ is incorporated and bound at the steps as it arrives from the gas phase.

Oxygen at the steps can have several consequences. It can effectively block a step site from further attachment of atoms because its bonds $(\mathrm{Ga}-\mathrm{O}-\mathrm{Al})$ would be satisfied. In addition, electronic interactions among oxygen, gallium, and arsenic atoms at the growth front may lead to large repulsive or attractive interactions. Repulsive interactions between the oxygen and other migrating charged species can increase the effective region over which sites for further growth will be blocked. Oxygen may additionally act as a kinetic barrier for the downward motion of atoms from the terraces as well as for the attachment of atoms at the step edges. It may impede the motion of other steps when these steps move during the step flow over the incorporated $\mathrm{O}$. The $\mathrm{O}$ may even pin these steps. Because the velocity of step segments containing the impurity atoms or moving past over the impurity atom incorporated in the layer underneath may be smaller compared to step segments for which there are no impurity interactions, step bunching may occur, resulting in periodic or quasiperiodic morphology. As the bulk concentration of impurities increases, impurities will incorporate more at the step edges resulting in a decrease in periodicity of the step bunching. At a very high impurity concentration, the effect of $\mathrm{O}$ is apparently sufficient to affect diffusion in the direction in which there was no step bunching to cause a breakdown of wavy morphology.

In summary, impurities adsorbed at the steps in MOVPE grown GaAs may act as a kinetic barrier to step flow and eventually lead to step bunching. The step bunching can lead to periodic or quasiperiodic morphology. The wavelength of the periodic morphology decreases with the increase in the impurity concentration. At a very high concentration of impurities, the periodic wavy surface morphology breaks into isolated small hillocks.

We would like to acknowledge discussions with Z. Y. Zhang, C. Teichert, and K.-C. Liu. This research was supported by the NSF through the Materials Research Group on Chemical Vapor Deposition (DMR-9121074 and DMR9414334) and the ARO (DAAH04-95-1-0322).

${ }^{1}$ M. Kasu and N. Kobayashi, Appl. Phys. Lett. 62, 1262 (1993).

${ }^{2}$ K. Hata, A. Kawazu, T. Okano, T. Ueda, and M. Akiyama, Appl. Phys. Lett. 63, 1625 (1993).

${ }^{3}$ I. Ishizaki, S. Goto, M. Kishiyada, T. Fukui, and H. Hasegawa, Jpn. J. Appl. Phys. 33, 721 (1994).

${ }^{4}$ C. C. Hsu, J. B. Xu, and I. H. Wilson, Appl. Phys. Lett. 64, 2105 (1994).

${ }^{5}$ S. Skala, S. T. Chou, K.-Y. Cheng, J. R. Tucker, and J. W. Lyding, Appl. Phys. Lett. 65, 722 (1994).

${ }^{6}$ E. J. Heller and M. G. Lagally, Appl. Phys. Lett. 60, 2675 (1992).

${ }^{7}$ C. Orme, M. D. Johnson, J. L. Sudijono, K. T. Leung, and B. G. Orr, Appl. Phys. Lett. 64, 860 (1994).

${ }^{8}$ G. W. Smith, A. J. Pidduck, C. R. Whitehouse, J. L. Glasper, A. M. Kier, and C. Pickering, Appl. Phys. Lett. 59, 3282 (1991).

${ }^{9}$ For an overview see, B. Lewis and J. C. Anderson, Nucleation and Growth in Thin Films (Academic, New York, 1978).

${ }^{10}$ D. E. Savage, E. J. Heller, Y.-H. Phang, M. Schacht, and M. G. Lagally, in Surface Disordering: Growth, Roughening and Phase Transitions, edited by R. Jullian, J. Kertisz, P. Meakin, and D. E. Wolf (Nova Science, Commack, NY, 1992).

${ }^{11}$ Z. Zhang, J. Deutch, and H. Metiu, Phys. Rev. B 48, 4972 (1993).

${ }^{12}$ T. F. Kuech, D. J. Wolford, E. Veuhoff, V. Deline, P. M. Mooney, R. Potemski, and J. Bradley, J. Appl. Phys. 62, 632 (1987).

${ }^{13}$ J. W. Huang, D. F. Gaines, T. F. Kuech, R. M. Potemski, and F. Cardone, J. Electron. Mater. 23, 659 (1994).

${ }^{14}$ T. Achtnich, G. Buri, and M. Ilegems, J. Vac. Sci. Technol. A 7, 2532 (1989).

${ }^{15}$ S. Nayak, J. M. Redwing, J. W. Huang, M. G. Lagally, and T. F. Kuech (unpublished). 